APJ IN PRESS

Preprint typeset using $\mathrm{LATEX}_{\mathrm{E}}$ style emulateapj v. 11/26/04

\title{
GRAVITATIONAL STABILITY OF CIRCUMNUCLEAR DISKS IN ELLIPTICAL GALAXIES
}

\author{
Daisuke Kawata ${ }^{1,2}$, Renyue $\mathrm{Cen}^{3}$, and Luis C. Ho ${ }^{1}$ \\ ApJ in press
}

\begin{abstract}
A significant fraction of nearby elliptical galaxies are known to have high-density gas disks in their circumnuclear $(\mathrm{CN})$ region $(0.1$ to a few $\mathrm{kpc})$. Yet, ellipticals, especially luminous ones, show little signs of recent star formation. To investigate the possible cause of the dearth of star formation in these systems, we study the gravitational stability of CN gas disks embedded within the gravitational potentials of both the stellar bulge component and the central massive black hole (BH) in elliptical galaxies. We find that $\mathrm{CN}$ disks in higher mass galaxies are generally more stable than those in lower mass galaxies, because higher mass galaxies tend to have more massive BHs and more centrally concentrated stellar density profiles. We also consider the case in which the central stellar density profile has a core, which is often observed for ellipticals whose total stellar mass is higher than about $10^{11} M_{\odot}$. Such a cored stellar density profile leads to more unstable CN disks than the power-law stellar density profile characteristic of less massive galaxies. However, the more massive BHs in highmass galaxies act to stabilize the $\mathrm{CN}$ disk. Our results demonstrate that the gravitational potentials of both the central BH and the stellar component should be taken into account when studying the properties of $\mathrm{CN}$ disks, as their stability is sensitive to both the $\mathrm{BH}$ mass and the stellar density profile. Our results could explain the observed trend that less luminous elliptical galaxies have a greater tendency to exhibit ongoing star formation than giant ellipticals.

Subject headings: galaxies: kinematics and dynamics - galaxies: formation - galaxies: stellar content
\end{abstract}

\section{INTRODUCTION}

Elliptical galaxies were believed to be completely dormant and therefore to consist of old stellar populations, due to the galactic wind which had blown the gas out and stopped star formation (Johnson \& Axford 1971; Mathews \& Baker 1971; Faber \& Gallagher 1976). The discovery of an X-ray-emitting hot interstellar medium (ISM) in these objects has dramatically renewed our view of their physical properties and formation history (e.g., Forman et al. 1979, 1985). Warm gas is also detected in a significant fraction of ellipticals $(55 \%-60 \%)$, although the estimated mass is small $\left(10^{3}-10^{4} M_{\odot}\right)$ (Phillips et al. 1986; Ho et al. 1997a). In addition, multiwavelength observations from optical to radio reveal the existence of the fair amount of H I gas (e.g., Knapp et al. 1985; Bregman et al. 1992; Huchtmeier 1994; Knapp 1999; Morganti et al. 2006), dust (e.g., Knapp et al. 1989; van Dokkum \& Franx 1995; Wiklind \& Henkel 1995; Tomita et al. 2000; Colbert et al. 2001; Tran et al. 2001; Martel et al. 2004), and molecular gas (e.g., Wiklind et al. 1995; Knapp \& Rupen 1996; Vila-Vilaró et al. 2003; Young 2005; Nakanishi et al. 2007) in a significant fraction of ellipticals. Particularly, some of the cold gas appears as a disk at the center of the galaxies. High-resolution images with $\mathrm{Hub}$ ble Space Telescope (HST) uncovered that the majority of elliptical galaxies possess such dusty cold disks, with a typical mass of $10^{4}-10^{6} M_{\odot}$ and sizes of $100-500 \mathrm{pc}$ (e.g., Tomita et al. 2000). Molecular gas disks are also observed in the central region, with estimated masses of $10^{6}-10^{9} M_{\odot}$ and extents up to a few kpc (Knapp 1999;

\footnotetext{
1 The Observatories of the Carnegie Institution of Washington, 813 Santa Barbara Street, Pasadena, CA 91101

2 Swinburne University of Technology, Hawthorn VIC 3122, Australia

${ }^{3}$ Department of Astrophysical Sciences, Princeton University, Peyton Hall, Ivy Lane, Princeton, NJ 08544
}

Okuda et al. 2005; Young 2005; Nakanishi et al. 2007). In this paper we call such observed central cold structures "circumnuclear" (CN) disks. So far, there is no evidence of a correlation between the mass of the $\mathrm{CN}$ disk and the total stellar mass of ellipticals (e.g., van Dokkum \& Franx 1995).

The estimated densities of the $\mathrm{CN}$ disks are relatively high. CN disks in gas-rich disk galaxies obviously harbor star formation (e.g., Phillips et al. 1986; Ho et al. 1997b; Koda et al. 2005). However, it is still a matter of debate whether or not the $\mathrm{CN}$ disks in ellipticals have star formation. Ho et al. (1997a, 2003) and Ho (2005) suggest that there is no clear evidence of star formation in the central region of bright elliptical galaxies. On the other hand, Phillips et al. (1986) show that less luminous ellipticals tend to have star formation. It also seems that the properties of the ISM of ellipticals depend on galaxy mass: the detection rates of H I and molecular gas are higher in less luminous galaxies (Lake \& Schommer 1984; Lees et al. 1991; Sadler et al. 2000; Sage et al. 2007). Okuda et al. (2005) measured the density and rotation of the $\mathrm{CN}$ disk in the radio galaxy $3 \mathrm{C} 31$, and suggest that its disk is stable against local gravitational instability, consistent with the lack of evidence for star formation in this system (Owen et al. 1990). Young (2005) also performed a similar analysis for NGC 83 and NGC 2320. Therefore, the gravitational stability of the disk may be a key factor for the star formation condition in the CN disk (Kennicutt 1989) (but see also Koda et al. 2005).

In the central region of elliptical galaxies, there are two empirical trends as a function of their mass. One is a well-established correlation between the black hole (BH) mass and the stellar mass of bulge (Magorrian et al. 1998; Marconi \& Hunt 2003; Häring \& Rix 2004). The other one is the fact that the central stellar density profile loosely depends on the mass of galaxies (Faber et al. 
1997; Ravindranath et al. 2001; Stiavelli et al. 2001; Ferrarese et al. 2006; Lauer et al. 2007). The high-resolution images from the HST enable the measurement of the stellar density profile in the central region of galaxies (Lauer et al. 1991, 1992b,a, 1993; Crane et al. 1993; Grillmair et al. 1994; Jaffe et al. 1994; Lauer et al. 1995). Lauer et al. (1995) suggest two different categories for the inner profile of elliptical galaxies: luminous galaxies have a "core" and less luminous galaxies have a "power-law" profile without any evidence of a core. On the other hand, Ravindranath et al. (2001) and Rest et al. (2001) found galaxies with "intermediate" inner profiles that cannot be categorized as either cores or power laws. Trujillo et al. (2004b) attempt to link the central and global profiles of early-type galaxies by noting that the outer profiles of ellipticals are well known to be fitted by the Sérsic law $\left(r^{1 / n}\right.$ law Sérsic 1968), and whose index $n$ is well-correlated with the global properties of galaxies, such as the effective radius and the total luminosity (e.g., Caon et al. 1993; Graham \& Guzmán 2003). They suggest that the inner power-law profile can be explained as the extension of the outer Sérsic profile, except for galaxies with central cores. Therefore, among power-law galaxies, the brighter members have profiles with larger $n$, and hence steeper power-law slope for the inner profile. On the other hand, Trujillo et al. (2004b) propose that luminous galaxies with cores can be described with a "core-Sérsic" profile, which is a combination of a core, approximated as an inner power-law profile with a shallow slope, and an outer Sérsic profile.

Motivated by these facts, we study the gravitational stability of the CN disk for elliptical galaxies with different masses. The stability of a rotating disk can be described by the so-called Toomre's $Q$-parameter (Toomre 1964; Goldreich \& Lynden-Bell 1965). This parameter also provides a criterion for star formation in rotating disks (e.g., Kennicutt 1989). Tan \& Blackman (2005) study the stability of the $\mathrm{CN}$ disk analytically. They conclude that $\mathrm{CN}$ disks are inevitably unstable, and should have star formation activity even in giant ellipticals, such as M87. However, so far, no indication of star formation has been observed in M87. Although Tan et al. (2007) report the detection of the molecular gas in the central region of M87, the optical spectral features of M87's CN disk are not consistent with star formation. The nuclear spectrum of M87 shows classical signatures of lowionization nuclear emission-line regions (Ho et al. 1997c), which as a group are consistent with being active galactic nuclei (AGNs) accreting at a low rate (Ho et al. 2003; Ho 2004).

The gravitational potential in the $\mathrm{CN}$ region is governed by the central $\mathrm{BH}$ as well as the stars in the inner bulge. The study of Tan \& Blackman (2005), however, ignores the potential of the stellar bulge component. Our analysis of the stability of the CN disk in ellipticals takes into account both contributions to the potential and their dependence on the global mass of the system. Section 2 summarizes our method to analyze the stability and describes models of the CN disk and the stellar potential in the CN region. Section 3 shows the results, and in Section 4 we discuss how our results fit with the recent observed properties of the central region of ellipticals.

\section{METHOD}

The stability of a self-gravitating disk can be analyzed by Toomre's $Q$-parameter (Toomre 1964; Goldreich \& Lynden-Bell 1965). The definition of Toomre's $Q$ parameter is

$$
Q \equiv \frac{c_{\mathrm{s}} \kappa}{\pi G \Sigma_{\mathrm{d}}}
$$

where $c_{\mathrm{s}}, \kappa$, and $\Sigma_{\mathrm{d}}$ are the sound velocity, epicycle frequency, and surface density of the gas disk. If $Q<1$, the disk is unstable. The specific frequency for a disk is described as

$$
\kappa^{2}=R \frac{d \Omega^{2}}{d R}+4 \Omega^{2},
$$

where $\Omega$ is the circular frequency.

We assume that the disk is steady, i.e. the accretion rate is the same at different radii. The accretion rate can be written as

$$
\dot{M}(R)=-2 \pi R \alpha_{\text {acc }} c_{\mathrm{s}}\left[\Omega(R)^{\prime} / \Omega(R)^{2}\right] \Sigma_{\mathrm{d}}(R)=\text { const. }
$$

where $\alpha_{\text {acc }}$ is the dimensionless viscosity parameter (Shakura \& Sunyaev 1973; Pringle 1981; Frank et al. 2002). This allows us to derive the density profile of the $\mathrm{CN}$ disk, $\Sigma_{\mathrm{d}}(R)$, once the total mass, $M_{\mathrm{d}}$, and the radius, $R_{\mathrm{d}}$, of the disk are fixed. The circular density profile $\Omega(R)$ is determined by the gravitational potential. For simplicity, we assume that the gravitational potential is dominated by the central $\mathrm{BH}$ and the stellar component, and that the contribution from the $\mathrm{CN}$ disk is negligible. As will be shown in Figure 1 below, this assumption is valid, except for the cases of massive and compact $\mathrm{CN}$ disk in smaller mass galaxies, which are inevitably unstable. We also assume that the stellar density is much higher than the dark matter density in the central region.

We examine the stability of the $\mathrm{CN}$ disk within the potential of the central region of spherical galaxies with different total masses of the stellar bulge, $M_{\mathrm{s}}$. We adopt the relation between the $\mathrm{BH}$ mass and the stellar mass from Häring \& Rix (2004),

$$
\log \left(M_{\mathrm{BH}} / M_{\odot}\right)=8.20+1.12 \log \left(M_{\mathrm{s}} / 10^{11} M_{\odot}\right) .
$$

Hence, once $M_{\mathrm{s}}$ is fixed, we can calculate the potential from the $\mathrm{BH}$ with the mass of $M_{\mathrm{BH}}$.

As mentioned in Section 1, the mass dependence of the inner stellar density profile is discussed by a number of authors. The first thorough study was carried out by the "Nuker team," who introduced the "Nuker law" to describe the observed surface brightness profile of the central regions of galaxies. The Nuker law (Lauer et al. 1995; Faber et al. 1997) is given by

$$
I(R)=I_{\mathrm{b}} 2^{(\beta-\gamma) / \alpha}\left(R / R_{\mathrm{b}}\right)^{-\gamma}\left[1+\left(R / R_{\mathrm{b}}\right)^{\alpha}\right]^{(\gamma-\beta) / \alpha} .
$$

The asymptotic logarithmic slope inside $R_{\mathrm{b}}$ is $\gamma$; the asymptotic logarithmic outer slope is $\beta$; and $\alpha$ parametrizes the sharpness of the break. The break radius, $R_{\mathrm{b}}$, is the point of maximum curvature in log-log coordinates. The break surface brightness, $I_{\mathrm{b}}$, is the surface brightness at $R_{\mathrm{b}}$. This function is designed to fit the surface brightness profile in the inner region, and not to describe the entire profile. Graham et al. (2003) proposed a new formula that is a combination of an inner power-law profile and an outer Sérsic law. They call it the "core-Sérsic law," which is described as

$$
I(R)=I^{\prime}\left[1+\left(R_{\mathrm{b}} / R\right)^{\alpha}\right]^{\gamma / \alpha}
$$




$$
\exp \left\{-b\left[\left(R^{\alpha}+R_{\mathrm{b}}^{\alpha}\right) / R_{\mathrm{e}}^{\alpha}\right]^{1 /\left(n_{\mathrm{s}} \alpha\right)}\right\}
$$

with

$$
I^{\prime}=I_{\mathrm{b}} 2^{-\gamma / \alpha} \exp \left[b 2^{1 /\left(n_{\mathrm{s}} \alpha\right)}\left(R_{\mathrm{b}} / R_{\mathrm{e}}\right)^{1 / n_{\mathrm{s}}}\right] .
$$

The parameter $R_{\mathrm{b}}$ is the break radius to separate the inner power law with a slope of $\gamma$ from the outer Sérsic law with effective radius $R_{\mathrm{e}}$ and index $n_{\mathrm{s}}$, and $I_{\mathrm{b}}$ is the surface brightness at $R_{\mathrm{b}}$. The parameter $\alpha$ controls the sharpness of the transition between the inner and outer profiles, where a higher value leads to sharper transitions. Trujillo et al. (2004b) suggest that the core-Sérsic law can be simplified with $\alpha \rightarrow \infty$ and still provide a good description to the observed profiles (see also Ferrarese et al. 2006). In this limit,

$$
\begin{aligned}
I(R)= & I_{\mathrm{b}}\left[\left(R_{\mathrm{b}} / R\right)^{\gamma} u\left(R_{\mathrm{b}}-R\right)\right. \\
& \left.+e^{b\left(R_{\mathrm{b}} / R_{\mathrm{e}}\right)^{1 / n_{\mathrm{s}}}} e^{-b\left(R / R_{\mathrm{e}}\right)^{1 / n_{\mathrm{s}}}} u\left(R-R_{\mathrm{b}}\right)\right],
\end{aligned}
$$

where $u(x-a)$ is the Heaviside step function.

It is still controversial which of the two formalisms better describes the surface brightness profile in the central region of galaxies (Ferrarese et al. 2006; Lauer et al. 2007). In this paper, we adopt the core-Sérsic law simply for computational convenience to link the density profiles in the inner region to the total mass of the stellar bulge. Although the simplified version of the core-Sérsic law as given in equation (8) has been adopted to fit recent observations (Trujillo et al. 2004b; Ferrarese et al. 2006), we use equation (6) by setting $\alpha$ to 5.0, for computational convenience. Lauer et al. (2007) demonstrate how the sharp transition generated from equation (8) leads to poor fits to the observed surface brightness profiles. The adopted $\alpha$ provides a less sharp transition. This assumption also guarantees that the deprojected density profile does not increase with radius with our adopted parameters (see below), but the profiles is still close to the simplified formula of equation (8) used in the observations.

As a comparison between the Nuker law (eq. 5) and the core-Sérsic law (eq. 6), we briefly mention how the logarithmic gradient, $\gamma^{\prime}\left(R^{\prime}\right)$, at radius of $R^{\prime}$ can be described for each fitting function (see also Trujillo et al. 2004b). Rest et al. (2001) show that for the Nuker law

$$
\gamma^{\prime}\left(R^{\prime}\right) \equiv-\left[\frac{d \log I(R)}{d \log R}\right]_{R^{\prime}}=\frac{\gamma+\beta\left(R^{\prime} / R_{\mathrm{b}}\right)^{\alpha}}{1+\left(R^{\prime} / R_{\mathrm{b}}\right)^{\alpha}}
$$

For the core-Sérsic law (eq. 6), Trujillo et al. (2004b) show that the slope can be written as

$\gamma^{\prime}\left(R^{\prime}\right)=\frac{b}{n_{\mathrm{s}}}\left(1 / R_{\mathrm{e}}\right)^{1 / n_{\mathrm{s}}} R^{\prime \alpha}\left(R^{\prime \alpha}+R_{\mathrm{b}}^{\alpha}\right)^{1 /\left(n_{\mathrm{s}} \alpha\right)-1}+\frac{\gamma\left(R_{\mathrm{b}} / R^{\prime}\right)^{\alpha}}{1+\left(R_{\mathrm{b}} / R^{\prime}\right)^{\alpha}}$

The core-Sérsic law (eq. 6) is equivalent to the Sérsic law, when $R_{\mathrm{b}}=0$ and $\gamma=0$. Then, the Sérsic law is described as

$$
I(x)=A_{\mathrm{s}} \exp \left(-b_{\mathrm{s}} x^{1 / n_{\mathrm{s}}}\right),
$$

with $x=R / R_{\mathrm{e}}$. Prugniel \& Simien (1997) derive the numerical solutions

$$
\begin{aligned}
& A_{\mathrm{s}}=I_{\text {tot }} \frac{b_{\mathrm{s}}^{2 n_{\mathrm{s}}}}{2 \pi n_{\mathrm{s}} \Gamma\left(2 n_{\mathrm{s}}\right)}, \\
& b_{\mathrm{s}}=2 n_{\mathrm{s}}-\frac{1}{3}+0.009876 / n_{\mathrm{s}},
\end{aligned}
$$

where $I_{\text {tot }}$ is the total bulge luminosity of the integration for $0 \leq x \leq \infty$ (see also Graham \& Driver 2005, and references therein). For simplicity, we define the total bulge mass, $M_{\mathrm{s}}$, as the total mass of the integration of the Sérsic law of equation (11), regardless of the existence of the core. Then, assuming a constant mass-to-luminosity ratio $\Upsilon$ at the different radii, we can describe the surface brightness at the break radius, $I_{\mathrm{b}}$, for the core-Sérsic law (eq. 6) as

$$
I_{\mathrm{b}}=\frac{M_{\mathrm{s}}}{\Upsilon} \frac{b_{\mathrm{s}}^{2 n_{\mathrm{s}}}}{2 \pi n_{\mathrm{s}} \Gamma\left(2 n_{\mathrm{s}}\right)} e^{-b_{\mathrm{s}}\left(R_{\mathrm{b}} / R_{\mathrm{e}}\right)^{1 / n_{\mathrm{s}}}} .
$$

It is well known that $R_{\mathrm{e}}$ and $n_{\mathrm{s}}$ correlate with the total luminosity of galaxies (e.g., Kormendy 1977; Caon et al. 1993; Graham 2002). From their fits of HST images of Virgo early-type galaxies using equation (8), Ferrarese et al. (2006) find that the profiles of bright galaxies $\left(M_{B} \leq-20.5 \mathrm{mag}\right)$ require a core, while those of less luminous galaxies can be described with the Sérsic law (see also Graham \& Guzmán 2003). They also derive the following relations between the $g$-band effective radius and $B$-band absolute magnitude:

$$
\log R_{\mathrm{e}}=-0.055\left(M_{B}+18\right)+1.14
$$

for the Sérsic galaxies, and

$$
\log R_{\mathrm{e}}=-0.22\left(M_{B}+18\right)+1.5
$$

for the core-Sérsic galaxies (Ferrarese et al. 2006, for error estimates, see). The units of $R_{\mathrm{e}}$ is arcseconds, which for Virgo corresponds to $1^{\prime \prime}=80.1$ pc. In addition, they suggest that the Sérsic index $n_{\mathrm{s}}$ and the $B$-band absolute magnitude are related by

$$
\log n_{\mathrm{s}}=-0.10\left(M_{B}+18\right)+0.39,
$$

valid for galaxies of both classes. In this paper we adopt these relations for computational convenience. Note that these relations are still not well established. For example, Graham \& Guzmán (2003) and Graham et al. (2006) suggest a curved relation between the luminosity and effective radius rather than two power laws.

We further assume that the stellar mass-to-light ratio for early-type galaxies in the $B$-band is $\Upsilon_{B}=$ $M_{\mathrm{s}} /\left(L_{B} / L_{B, \odot}\right)=7$, with $M_{B, \odot}=5.48 \mathrm{mag}$, and that $\Upsilon_{B}$ is independent of mass; these assumptions are sufficiently accurate for early-type galaxies (Trujillo et al. 2004a). With some exceptions, Ferrarese et al. (2006) find that there is a critical luminosity that separates the Sérsic galaxies from the core-Sérsic galaxies. With the assumptions made above, their critical luminosity of $M_{B, \mathrm{c}}=-20.5 \mathrm{mag}$ corresponds to $M_{\mathrm{s}, \mathrm{c}}=1.73 \times 10^{11}$ $M_{\odot} ;$ systems with masses lower than $M_{\mathrm{s}, \mathrm{c}}$ are assumed to follow the Sérsic law. Then, we derive the half-mass radius using equations (15) and (16), with the assumption that the $g$-band half-light radius is similar to the halfmass radius. Once we fix $M_{\mathrm{s}}$, all the parameters of equation (6) can be determined, and equation (6) provides the projected mass density profile of $\Sigma_{\mathrm{s}}(R)=I_{B}(R) \Upsilon_{B}$. Assuming spherical symmetry, we can derive the threedimensional stellar mass density $\rho_{\mathrm{s}}(r)$ through (e.g., Binney \& Tremaine 1987)

$$
\rho_{\mathrm{s}}(r)=-\frac{1}{\pi} \int_{r}^{\infty} \frac{d \Sigma_{\mathrm{s}}(R)}{d R} \frac{d R}{\sqrt{R^{2}-r^{2}}} .
$$

The analytical formula of three-dimensional stellar density profiles is also discussed in Prugniel \& Simien (1997), Mazure \& Capelato (2002), Terzić \& Graham (2005), and references therein. 


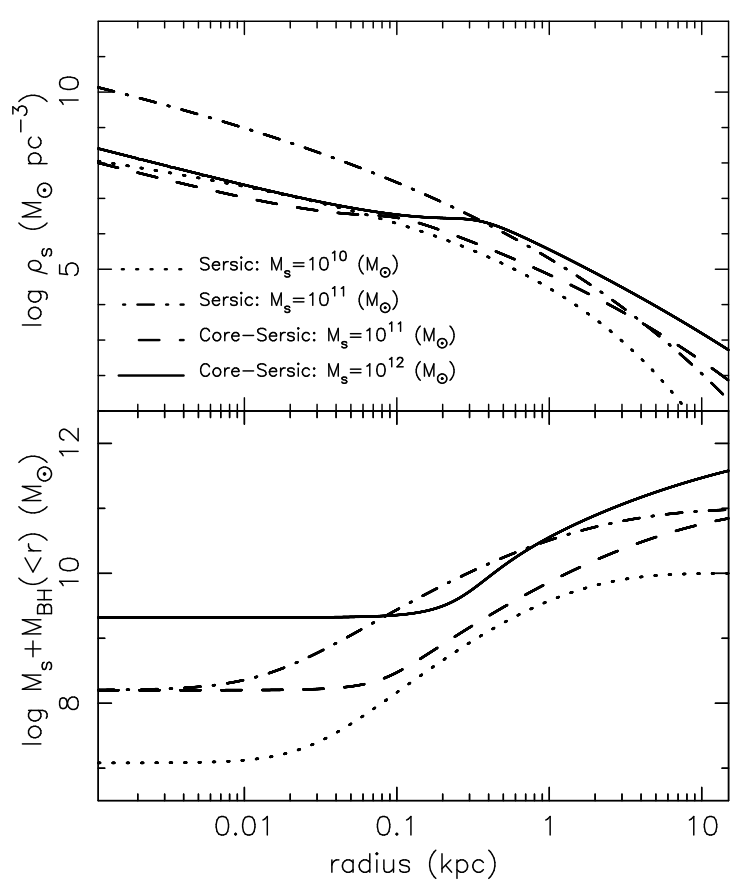

FIG. 1.- Stellar density profile as a function of three-dimensional radius (top) and the total mass of stars and $\mathrm{BH}$ within the radius (bottom) for four different models indicated in the upper panel.

TABLE 1

Stellar Bulge Density Profiles for the Host Galaxies

\begin{tabular}{cccccccc}
\hline \hline Model & $\begin{array}{c}M_{\mathrm{s}} \\
\left(M_{\odot}\right)\end{array}$ & Profile & $\begin{array}{c}R_{\mathrm{e}} \\
(\mathrm{kpc})\end{array}$ & $n_{\mathrm{S}}$ & $\begin{array}{c}R_{\mathrm{b}} \\
(\mathrm{pc})\end{array}$ & $\gamma$ & $\begin{array}{c}M_{B}{ }^{\mathrm{a}} \\
(\mathrm{mag})\end{array}$ \\
\hline $\mathrm{S} 10$ & $10^{10}$ & Sérsic & 1.03 & 2.14 & - & - & -17.4 \\
$\mathrm{~S} 11$ & $10^{11}$ & Sérsic & 1.41 & 3.81 & - & - & -19.9 \\
$\mathrm{cS} 11$ & $10^{11}$ & core-Sérsic & 6.66 & 3.81 & 9.32 & 0.1 & -19.9 \\
$\mathrm{cS} 12$ & $10^{12}$ & core-Sérsic & 23.6 & 6.77 & 331 & 0.1 & -22.4 \\
\hline
\end{tabular}

${ }^{a} B$-band luminosity estimated with a stellar mass-to-light ratio of $\Upsilon_{B}=M_{\mathrm{s}} /\left(L_{B} / L_{B, \odot}\right)=7$.

\section{RESULTS}

\subsection{Stability of CN Disks with Fixed Mass and Radius}

As mentioned in Section 1, so far no correlation has been found between the mass or size of CN disks and the host galaxy mass. In this section we study how the stability of $\mathrm{CN}$ disks with fixed mass and radius depends on the total mass of the host galaxies. Here, we focus on a disk with mass $\log \left(M_{\mathrm{d}} / \mathrm{M}_{\odot}\right)=8$ and radius $R_{\mathrm{d}}=2.5$ $\mathrm{kpc}$, which is roughly the same as the $\mathrm{CN}$ disk observed in NGC 4476 by Young (2002). Disks with a range of different masses and radii will be discussed in the next section. Note that once $M_{\mathrm{d}}$ and $R_{\mathrm{d}}$ are fixed, $\Sigma_{\mathrm{d}}(R)$ does not depend on $\alpha_{\text {acc }}$ or $c_{\mathrm{s}}$. Hence, $Q(R)$ described by equation (1) is independent of $\alpha_{\text {acc }}$ and is simply proportional to $c_{\mathrm{s}}=\sqrt{\gamma_{\mathrm{d}} k_{\mathrm{B}} T_{\mathrm{d}} /\left(\mu m_{\mathrm{p}}\right)}$, where $\gamma_{\mathrm{d}}, k_{\mathrm{B}}, T_{\mathrm{d}}$, $\mu$, and $m_{\mathrm{p}}$ are the specific heat, Boltzmann's constant, the gas disk temperature, the mean molecular mass, and the proton mass, respectively. Throughout the paper, we fixed $c_{\mathrm{s}}$ to a value corresponding to $\mu=0.6, \gamma_{\mathrm{d}}=1$, and $T_{\mathrm{d}}=30 \mathrm{~K}$, which is a typical temperature of observed CN disks (e.g., Wiklind et al. 1995; Tran et al. 2001).

Stability of the CN disk is examined for host galaxy models with stellar bulge masses of $\log \left(M_{\mathrm{s}} / M_{\odot}\right)=10$,
11, and 12. We apply the Sérsic law to the model with $\log \left(M_{\mathrm{S}} / M_{\odot}\right)=10($ model S10), and the core-Sérsic law to the model with $\log \left(M_{\mathrm{s}} / M_{\odot}\right)=12(\operatorname{model} \mathrm{cS} 12)$. Since the adopted scaling relation used in Section 2 is known to have significant scatter and the model of $\log \left(M_{\mathrm{S}} / M_{\odot}\right)=$ 11 is close to the critical mass of $M_{\mathrm{s}, \mathrm{c}}=1.73 \times 10^{11} M_{\odot}$ for the two stellar density profiles, we consider two cases of the Sérsic (model S11) law and the core-Sérsic (model $\mathrm{cS11}$ ) laws for host galaxies with $\log \left(M_{\mathrm{S}} / M_{\odot}\right)=11$. All the parameter values for the stellar density profiles and the estimated $B$-band absolute magnitude are summarized in Table 1 . We choose $\gamma=0.1$ for the core-Sérsic law of equation (6), which is a relatively low value among observed cores (e.g., Ferrarese et al. 2006); this is a conservative choice because it leads to a maximally unstable CN disk.

Figure 1 shows that the stellar density profile and the total mass of stars and $\mathrm{BH}$ as a function of radius for the four models. Comparison between models S11 and S10 shows that the density profile is steeper for higher mass galaxies, which have a larger $n_{\mathrm{s}}$. The core-Sérsic models lead to a lower density in the inner region. Consequently, model cS12 has even lower density than model S11 and a similar density to model S10 within the break radius of $\mathrm{cS} 12$. However, higher mass galaxies have a more massive $\mathrm{BH}$. As a result, the total mass within a given radius is generally higher for the higher mass galaxies, except around the break radius for the core-Sérsic models.

Figure 2 presents the values of Toomre's $Q$-parameter at different radii of the $\mathrm{CN}$ disk for the four models. We also examine two additional cases for each model; one is the case assuming no $\mathrm{BH}$ (dotted line), and the other one (dashed line) ignores the stellar potential (i.e., only the Keplerian potential of the $\mathrm{BH}$ is considered). As described in Section 2, the circular velocity (Fig. 3) is calculated purely by the $\mathrm{BH}$ and/or stellar potential, and the gas disk density profile (Fig. 4) is derived from the condition of $\dot{M}(R)=$ constant in equation (3).

The BH-only potential results (dashed lines) demonstrate that since higher mass galaxies have a more massive $\mathrm{BH}, \mathrm{CN}$ disks are more stable in higher mass galaxies. However, for the assumed $\mathrm{CN}$ disk, even model cS12 still has $Q<1$-i.e., the $\mathrm{CN}$ disk is unstable. The stellar $+\mathrm{BH}$ potential results clearly demonstrate that the stellar potential greatly helps to stabilize the $\mathrm{CN}$ disk. Moreover, comparison between the results for the stellar $+\mathrm{BH}$ potential and the results of the case of stellaronly potential indicates that the $\mathrm{BH}$ helps to stabilize the $\mathrm{CN}$ disk only in the inner region (a few $100 \mathrm{pc}$, depending on the $\mathrm{BH}$ mass and the stellar density profiles). Therefore, the stellar potential cannot be ignored in studying the dynamics of the $\mathrm{CN}$ disk.

For the Sérsic models, the stellar potential stabilizes the $\mathrm{CN}$ disk more in the higher mass galaxies, because higher mass galaxies have larger $n_{\mathrm{s}}$, i.e. higher central concentration of the stellar potential (see also Fig. 1). At a fixed stellar mass of $\log \left(M_{\mathrm{s}} / M_{\odot}\right)=11$ the core-Sérsic model leads to a lower $Q$-value and a more unstable disk. The surface density profile of the $\mathrm{CN}$ disk is sensitive to this change of the stellar potential profile (Fig. 4). The stellar-only potential results clearly demonstrate that the disk gas density has a peak around the break radius. Due to this peak density, the $Q$-value becomes as low as the 


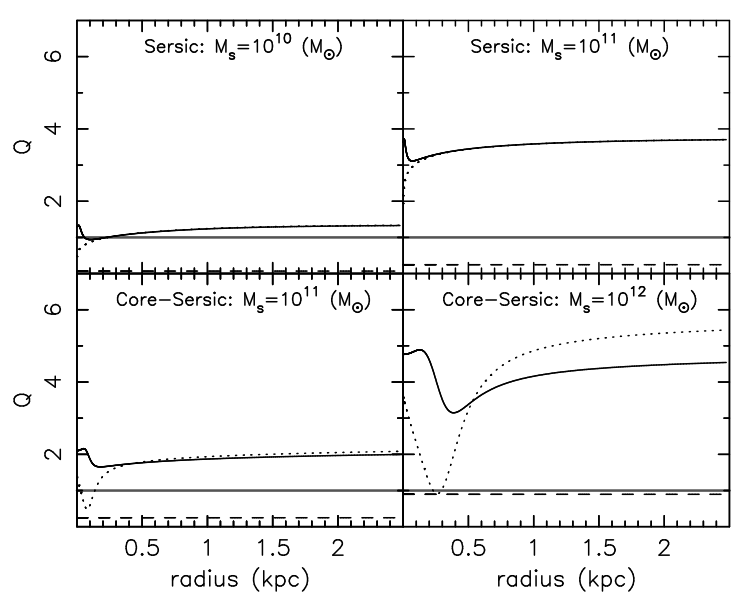

FIG. 2.- Toomre's $Q$-value as a function of radius for the $\mathrm{CN}$ disk with $M_{\mathrm{d}}=10^{8} M_{\odot}$ and $R_{\mathrm{d}}=2.5 \mathrm{kpc}$ in galaxy models indicated in the panels. The black solid line presents the $Q$-value calculated by taking into account both the $\mathrm{BH}$ and the stellar potential. The dotted line only includes the stellar potential, while the dashed line takes into account only the potential of the central $\mathrm{BH}$, which is correlated with the mass of the stellar component as assumed in equation (4). The gray solid line marks $Q=1$.

case of the BH-only potential. However, the BH can stabilize the disk in the inner region, and the stellar $+\mathrm{BH}$ potential case is much more stable. As a result, model cS11 is more stable than model S10.

Note that, as shown in Lauer et al. (2007), the Sérsic law often underestimates the surface brightness in the central region where a high-density stellar component is often seen. Ferrarese et al. (2006) invoke an additional component, which they call "nuclei," to fit this central high-density component. Although we do not include the nuclei in this study for simplicity, such a compact central component also stabilizes the $\mathrm{CN}$ disk in a similar way to the BH. Côté et al. (2006) find that the estimated mass of the nuclei is similar to what the $\mathrm{BH}$ mass and bulge mass relation, such as equation (4), predicts. Therefore, the effect of the nuclei would be similar to assuming a factor of two more massive $\mathrm{BH}$.

In general, $\mathrm{CN}$ disks in higher mass galaxies are more stabilized, due to higher mass $\mathrm{BH}$ and more centrally concentrated profile of the stellar component. However, if the host galaxy has the core-Sérsic law profile, the CN disk is more unstable, compared with the $\mathrm{CN}$ disk in a galaxy with the same stellar mass and $\mathrm{BH}$ mass, but having Sérsic profile.

\subsection{Stability of CN Disks with Various Masses and Radii}

In this section, we again consider the four models shown in Table 1, but study the stability of the CN disks with different masses and radii. Figure 5 shows the $Q$ value for the $\mathrm{CN}$ disks with radii in the range of $0.05-15$ kpc and masses spanning $\log \left(M_{\mathrm{d}} / M_{\odot}\right)=4-10$. Here, the figure shows the minimum $Q$-value in each model. For example, $Q_{\min } \approx 3.15$ at $r_{\mathrm{d}}=380 \mathrm{pc}$ for model cS12 in Figure 2.

As expected, CN disks with higher gas mass and smaller radii are more unstable. Comparison between S10 and S11 demonstrates that for two CN disks with the same mass and radius in a Sérsic-law galaxy, the disk in the higher mass galaxy is always more stable, due to the higher mass $\mathrm{BH}$ and the more centrally concentrated

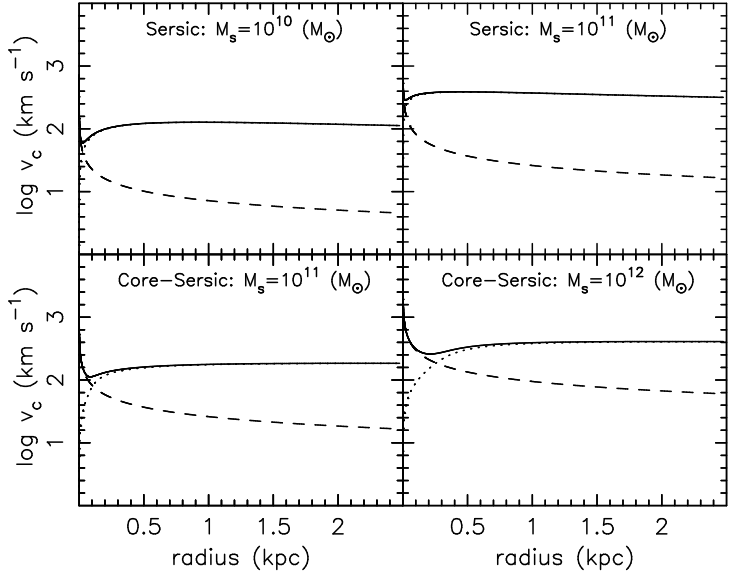

FIG. 3.- The circular velocity, $v_{\mathrm{c}}$, as a function of the radius of the CN disk with $M_{\mathrm{d}}=10^{8} M_{\odot}$ and $R_{\mathrm{d}}=2.5 \mathrm{kpc}$ in galaxy models indicated in the panels. The solid line takes into account both the $\mathrm{BH}$ and the stellar potential. The dotted line only includes the stellar potential, while the dashed line takes into account only the potential of the central $\mathrm{BH}$.

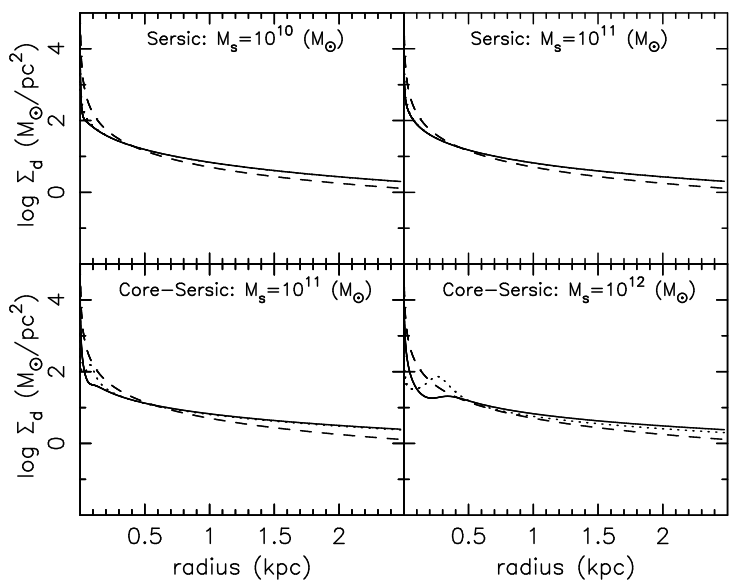

FIG. 4. - The surface density profile, $\Sigma_{\mathrm{d}}(R)$, of the CN disk with $M_{\mathrm{d}}=10^{8} M_{\odot}$ and $R_{\mathrm{d}}=2.5 \mathrm{kpc}$ in galaxy models indicated in the panels. The solid line takes into account both the BH and the stellar potential. The dotted line only includes the stellar potential, while the dashed line takes into account only the potential of the central BH.

stellar density profile. If the $\mathrm{CN}$ disk is smaller than the break radius, the highest mass model (i.e. model $\mathrm{cS} 12$ ) is the most stable. For a $\mathrm{CN}$ disk with a size comparable to the break radius, model cS12 leads to a less stable condition than model S11, and for the larger CN disk model cS12 is as stable as model S11. Therefore, for large $\mathrm{CN}$ disks in high mass galaxies, the size of the disk with respect to the break radius of the host galaxies is an important factor for the stability.

\section{DISCUSSION AND CONCLUSIONS}

Our analysis of the stability of CN disks reveals the following trends.

(1) The CN disk is stabilized by the presence of the central BH. Since higher mass galaxies tend to have more massive $\mathrm{BHs}$, the $\mathrm{CN}$ disk is more stable in higher mass galaxies in general.

(2) The stellar potential is also important for the stability of the CN disk. 


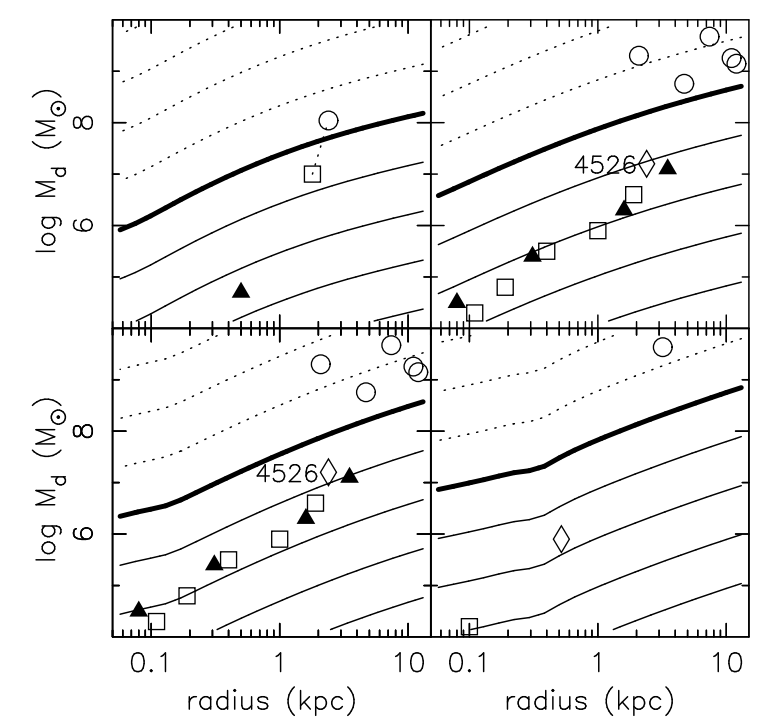

FIG. 5.- The minimum $Q$-value for the $\mathrm{CN}$ disk with different masses and radii. The upper-left, upper-right, lower-left, and lower-right panels show the results of model S10, S11, cS11, and cS12, respectively. The thick solid line indicates $Q=1$. The dotted lines and thin solid lines correspond to contours for $Q<1$ and $Q>1$. The levels are separated by $\delta \log Q=1.0$. Open circles denote CN disks observed in CO emission by Young $(2002,2005)$. The rest of the symbols show the mass and size of the CN disk estimated from the optical color excess of dusty disks observed with $H S T$ (Tomita et al. 2000). Solid triangles and open squares correspond to the galaxies whose profile has been fitted with a Sérsic law and a core-Sérsic law, respectively (Ferrarese et al. 2006; Lauer et al. 2007); open diamonds mark galaxies with unclassified profiles. NGC 4476, which has been observed both in CO (Young 2002) and with HST (Tomita et al. 2000), is connected with a dotted line.

(3) For Sérsic-law galaxies, the CN disk is more stable in higher mass galaxies because they tend to have more centrally concentrated stellar density profiles.

(4) The existence of a central stellar core in luminous ellipticals makes the CN disk unstable, especially around the break radius.

As discussed in Section 2, the central surface brightness profiles for relatively low-mass galaxies $\left(M_{\mathrm{s}} \leq 10^{11}\right.$ $M_{\odot}$ ) are generally described by the Sérsic law, with the trend of higher mass galaxies tending to have larger $n_{\mathrm{s}}$. Points (1)-(3) above indicate that CN disks in lower mass galaxies are more unstable. On the other hand, relatively high-mass galaxies $\left(\log \left(M_{\mathrm{s}} / M_{\odot}\right) \geq 11\right)$ tend to have stellar density profiles that contain a central core, which leads to a more unstable CN disk compared to the Sérsic law. On the other hand, since such systems also have more massive $\mathrm{BHs}, \mathrm{CN}$ disks in higher mass galaxies are kept stable, especially within the break radius. These trends can explain the observational trends outlined in Section 1. Because CN disks are more stable in more luminous ellipticals, this provides a natural explanation for central star formation to be curtailed in giant ellipticals, whereas less luminous ellipticals apparently have host nuclear star formation with greater ease, even though cold ISM in the form of dusty nuclear disks are observed to be just as prevalent in both environments.

It is also worth stressing that, as seen in Figure 4, the $\mathrm{CN}$ disk can remain stable even if the density of the disk is more than $100 M_{\odot} \mathrm{pc}^{-2}$ within $100 \mathrm{pc}$. This is because the $\mathrm{BH}$ stabilizes the $\mathrm{CN}$ disk in the central region, as seen in Figure 2. This density is much higher than the canonical density threshold for star formation suggested by both observations (Kennicutt 1989) and theory (e.g., Schaye 2004), $\Sigma_{\text {th }} \sim 3-10 M_{\odot} \mathrm{pc}^{-2}$. Our stability analysis offers a simple explanation for the lack of star formation (e.g., Okuda et al. 2005; Young 2005) in CN disks that otherwise have high densities.

At a fixed total stellar mass for the host galaxy, galaxies with higher $n_{\mathrm{s}}$ Sérsic law have more stable CN disks, while the core-Sérsic law leads to more unstable CN disks than the Sérsic law. Therefore, the frequency of central star formation activity should depend on both the central stellar density profile as well as the BH mass. Recent near-ultraviolet observations performed with Galaxy Evolution Explorer (GALEX) indicate that some fraction of early-type galaxies have a small amount of recent star formation (Yi et al. 2005). Based on semi-analytic model predictions Schawinski et al. (2006) demonstrate that the observed near-ultraviolet color distributions of early-type galaxies can be explained if there is a critical BH mass at the fixed velocity dispersion for galaxies that have recent star formation. They suggested that the critical BH mass likely comes from strong AGN heating created by the massive BH (e.g., Binney \& Tabor 1995; Silk \& Rees 1998; Kawata \& Gibson 2005; Springel et al. 2005; Croton et al. 2006; Bower et al. 2006; Ciotti \& Ostriker 2007). However, our study demonstrates that the existence of a critical condition for star formation at the fixed velocity dispersion can be due to the stability of the CN disk, which is governed by both the stellar density profile and the $\mathrm{BH}$ mass.

As mentioned in Section 1, the size and mass of the CN disk have been measured by several observational studies. Some of these measurements are plotted in Figure 5 to compare with our model predictions. The circles in Figure 5 correspond to objects whose CN disk mass and radius were estimated from $\mathrm{CO}$ emission observed with interferometers in Young $(2002,2005)$. Figure 5 also contains objects whose disk properties were estimated from the color excess of dust features measured in optical images of the central region of early-type galaxies observed with the HST (Tomita et al. 2000) ${ }^{4}$. The stellar density profiles of the central region for the majority of the galaxies in Tomita et al. (2000) have been studied by Ferrarese et al. (2006) and/or Lauer et al. (2007). This allows us to further distinguish the objects by their central profile type.

Following Section 2, we estimated the stellar mass from the $B$-band luminosity, assuming a stellar massto-light ratio of $\Upsilon_{B}=M_{\mathrm{s}} /\left(L_{B} / L_{B, \odot}\right)=7$. The $B$-band luminosities come from the NASA/IPAC Extragalactic Database (NED). We plot the data for galaxies with masses $\log \left(M_{\mathrm{S}} / M_{\odot}\right)<10.5$ and $\log \left(M_{\mathrm{S}} / M_{\odot}\right)>11.5$ in the upper-left and lower-right panels of the figure, respectively; the rest of galaxies are shown in both the upper-right and lower-left panels.

Only one object-NGC 4476 - has been measured with both techniques (CO from Young (2002) and dust from Tomita et al. (2000)); the two data points are connected with the dotted line. Young (2002) obtained a much higher gas mass of $1.1 \times 10^{8} M_{\odot}$ within $2.4 \mathrm{kpc}$,

4 Tomita et al. (2000) find that some galaxies in their sample have irregular dust morphology. Figure 5 only shows data for galaxies whose dust morphology is classified a disk. 
compared with a mass of $1.0 \times 10^{7} M_{\odot}$ within $1.8 \mathrm{kpc}$ in Tomita et al. (2000). The order-of-magnitude discrepancy in mass cannot be explained by the slightly different distances adopted by these authors (18 Mpc by Young (2002) and $16.8 \mathrm{Mpc}$ by Tomita et al. (2000)). It is noteworthy that all of the CO-measured $\mathrm{CN}$ disks have systematically larger disk masses than the dust-measured systems, suggesting that at least part of the discrepancy may be due to systematic errors in the estimated gas masses. Both sets of observations require a conversion factor to arrive at a gas mass. In the case of the $\mathrm{CO}$ observations, a standard Galactic CO-to- $\mathrm{H}_{2}$ conversion factor was adopted. The CO-to- $\mathrm{H}_{2}$ conversion factor, however, may be systematically lower in regions of high metallicity (Arimoto et al. 1996), as is the case in the central regions of massive galaxies, a suggestion supported by radiative transfer calculations in numerical simulations of CN disks (Wada \& Tomisaka 2005). If this is the case, then the gas masses from Young (2002, 2005) are overestimated.

A similar caveat applies to the gas masses estimated from the dust extinction, which assume a Galactic gasto-dust mass ratio (Tomita et al. 2000). However, dust mass estimated from optical extinction should be considered as lower limit to the true dust masses (Goudfrooij \& de Jong 1995; Tran et al. 2001; Martel et al. 2004). For example, Goudfrooij \& de Jong (1995) show that the dust masses derived from the far-infrared emission are roughly an order of magnitude higher than those estimated from optical extinction alone. They argue that the optical extinction may underestimate the dust mass by about factor of 2 due to the assumption that the dust is in front of the stars (see also Martel et al. 2000, who claim that this effect is more significant). Goudfrooij \& de Jong (1995) also discuss that the discrepancy in the estimated dust mass is mainly due to the presence of diffusely distributed dust, which cannot be seen as optical extinction. We note, however, that such a diffuse component is unlikely to be present as a rotating disk, as it is more likely to be supported by velocity dispersion. In any case, it seems plausible that the gas masses derived from the optical dust features may be an underestimate of the true gas mass, thereby narrowing the disagreement with the CO-based masses. Until more accurate gas masses are available, it is difficult to draw more quantitative comparisons between our model predictions and observations.

To see if there is any sign of ongoing star formation in the sample of galaxies shown in Figure 5, we have done a careful search of the literature to inspect published optical spectra (Phillips et al. 1986; Bonatto et al. 1989; Ho et al. 1997c; Cretton et al. 2000; Donzelli \& Davoust 2003). Galaxies with ongoing star formation show optical emission-line ratios that are readily distinguishable from other sources of ionization (e.g., active galactic nuclei; see Ho et al. 1997c). Among all the objects, only NGC 4526 has a spectral classification consistent with stellar photoionization (Ho et al. 1997c). The rest either have no star formation (14 galaxies) or have insufficient spectral information to tell (5 galaxies). Although NGC 4526, which is highlighted in Figure 5 , is in the stable $(Q>1)$ region, interestingly, NGC 4526 has one of the most unstable $\mathrm{CN}$ disks among the $H S T$-measured sample. Also, none of the CO-measured $\mathrm{CN}$ disks, which are located in the unstable region in Figure 5, show a clear indication of star formation. Although this contradicts with our prediction, because of the above-mentioned ambiguities in the estimates of the gas masses from the observations, it is difficult to conclude if these galaxies require additional process to explain their suppression of star formation.

It is clear from inspection of Figure 5 that once the mass of the $\mathrm{CN}$ disk becomes high enough, the disk will inevitably become unstable for star formation, one of the consequences of which may be to aid gas fueling to the AGN (Wada 2004). This type of situation is likely to be realized in the aftermath of a gas-rich major merger, whereby the large amount of the gas dissipated toward the center can generate a high-mass CN disk. Highresolution millimeter observations of luminous infrared galaxies indeed suggest that systems with larger central gas surface densities tend to either form stars with greater efficiency or have a higher probability of hosting an AGN (Bryant \& Scoville 1999).

Finally, we speculate that star formation induced in an unstable CN disk may be directly related to the formation of kinematically decoupled cores (KDCs) that are often seen in the centers of early-type galaxies (e.g., Kormendy 1984; Bender 1988; Bender \& Surma 1992; Caon et al. 2000) as well as the central stellar disk that many power-law ellipticals seem to have (Lauer et al. 2005). One of the intriguing clues regarding the origin of KDCs is that large cores are always old (McDermid et al. 2006). If KDCs are a by-product of unstable CN disks, then large KDCs require large, massive $\mathrm{CN}$ disks, which probably can only be formed through major, gas-rich mergers, which are most prevalent at earlier epochs.

We thank Alister Graham, Tod Lauer, and Lisa Young for helpful comments. This research has made use of the NASA/IPAC Extragalactic Database (NED) which is operated by the Jet Propulsion Laboratory, California Institute of Technology, under contract with the National Aeronautics and Space Administration. This work is supported in part by grants NNG05GK10G and AST0507521. The work of L. C. H. is supported by the Carnegie Institution of Washington and by NASA grants from the Space Telescope Science Institute (operated by AURA, Inc., under NASA contract NAS5-26555).

\section{REFERENCES}

Arimoto, N., Sofue, Y., \& Tsujimoto, T. 1996, PASJ, 48, 275 Bender, R. 1988, A\&A, 202, L5

Bender, R., \& Surma, P. 1992, A\&A, 258, 250

Binney, J., \& Tabor, G. 1995, MNRAS, 276, 663

Binney, J., \& Tremaine, S. 1987, Galactic Dynamics (Princeton, NJ: Princeton University Press), 747

Bonatto, C., Bica, E., \& Alloin, D. 1989, A\&A, 226, 23
Bower, R. G., Benson, A. J., Malbon, R., Helly, J. C., Frenk, C. S., Baugh, C. M., Cole, S., \& Lacey, C. G. 2006, MNRAS, 370, 645 Bregman, J. N., Hogg, D. E., \& Roberts, M. S. 1992, ApJ, 387, 484 Bryant, P. M., \& Scoville, N. Z. 1999, AJ, 117, 2632

Caon, N., Capaccioli, M., \& D'Onofrio, M. 1993, MNRAS, 265, 1013

Caon, N., Macchetto, D., \& Pastoriza, M. 2000, ApJS, 127, 39

Ciotti, L., \& Ostriker, J. P. 2007, ApJ, in press 
Colbert, J. W., Mulchaey, J. S., \& Zabludoff, A. I. 2001, AJ, 121, 808

Côté, P., et al. 2006, ApJS, 165, 57

Crane, P., et al. 1993, AJ, 106, 1371

Cretton, N., Rix, H.-W., \& de Zeeuw, P. T. 2000, ApJ, 536, 319

Croton, D. J., et al. 2006, MNRAS, 365, 11

Donzelli, C. J., \& Davoust, E. 2003, A\&A, 409, 91

Faber, S. M., et al. 1997, AJ, 114, 1771

Faber, S. M., \& Gallagher, J. S. 1976, ApJ, 204, 365

Ferrarese, L., et al. 2006, ApJS, 164, 334

Forman, W., Jones, C., \& Tucker, W. 1985, ApJ, 293, 102

Forman, W., Schwarz, J., Jones, C., Liller, W., \& Fabian, A. C. 1979, ApJ, 234, L27

Frank, J., King, A., \& Raine, D. J. 2002, Accretion Power in Astrophysics: Third Edition (Cambridge, UK: Cambridge University Press)

Goldreich, P., \& Lynden-Bell, D. 1965, MNRAS, 130, 97

Goudfrooij, P., \& de Jong, T. 1995, A\&A, 298, 784

Graham, A. W. 2002, MNRAS, 334, 859

Graham, A. W., \& Driver, S. P. 2005, PASA, 22, 118

Graham, A. W., Erwin, P., Trujillo, I., \& Asensio Ramos, A. 2003 AJ, 125, 2951

Graham, A. W., \& Guzmán, R. 2003, AJ, 125, 2936

Graham, A. W., Merritt, D., Moore, B., Diemand, J., \& Terzić, B. 2006, AJ, 132, 2711

Grillmair, C. J., Faber, S. M., Lauer, T. R., Baum, W. A., Lynds, R. C., O'Neil, Jr., E. J., \& Shaya, E. J. 1994, AJ, 108, 102

Häring, N., \& Rix, H.-W. 2004, ApJ, 604, L89

Ho, L. C. 2004, in Carnegie Observatories Astrophysics Series, Vol. 1: Coevolution of Black Holes and Galaxies, ed. L. C. Ho (Cambridge: Cambridge Univ. Press), 292

- 2005, ApJ, 629, 680

Ho, L. C., Filippenko, A. V., \& Sargent, W. L. W. 1997a, ApJ, 487,568

- $1997 \mathrm{~b}$, ApJ, 487, 579

- 1997c, ApJS, 112, 315

—. 2003, ApJ, 583, 159

Huchtmeier, W. K. 1994, A\&A, 286, 389

Jaffe, W., Ford, H. C., O'Connell, R. W., van den Bosch, F. C., \& Ferrarese, L. 1994, AJ, 108, 1567

Johnson, H. E., \& Axford, W. I. 1971, ApJ, 165, 381

Kawata, D., \& Gibson, B. K. 2005, MNRAS, 358, L16

Kennicutt, R. C. 1989, ApJ, 344, 685

Knapp, G. R. 1999, in Star Formation in Early-Type Galaxies, ed. P. Carral \& J. Cepa (San Francisco: ASP), 119

Knapp, G. R., Guhathakurta, P., Kim, D.-W., \& Jura, M. A. 1989, ApJS, 70, 329

Knapp, G. R., \& Rupen, M. P. 1996, ApJ, 460, 271

Knapp, G. R., Turner, E. L., \& Cunniffe, P. E. 1985, AJ, 90, 454

Koda, J., Okuda, T., Nakanishi, K., Kohno, K., Ishizuki, S., Kuno, N., \& Okumura, S. K. 2005, A\&A, 431, 887

Kormendy, J. 1977, ApJ, 218, 333

- 1984, ApJ, 287, 577

Lake, G., \& Schommer, R. A. 1984, ApJ, 280, 107

Lauer, T. R., et al. 1991, ApJ, 369, L41

- 1992a, AJ, 104, 552

- 1992b, AJ, 103, 703

1993, AJ, 106, 1436

1995, AJ, 110, 2622

2005, AJ, 129, 2138

- 2007, ApJ, in press
Lees, J. F., Knapp, G. R., Rupen, M. P., \& Phillips, T. G. 1991, ApJ, 379, 177

Magorrian, J., et al. 1998, AJ, 115, 2285

Marconi, A., \& Hunt, L. K. 2003, ApJ, 589, L21

Martel, A. R., et al. 2004, AJ, 128, 2758

Martel, A. R., Turner, N. J., Sparks, W. B., \& Baum, S. A. 2000, ApJS, 130, 267

Mathews, W. G., \& Baker, J. C. 1971, ApJ, 170, 241

Mazure, A., \& Capelato, H. V. 2002, A\&A, 383, 384

McDermid, R. M., et al. 2006, MNRAS, 373, 906

Morganti, R., et al. 2006, MNRAS, 371, 157

Nakanishi, H., Tosaki, T., Kohno, K., Sofue, Y., \& Kuno, N. 2007, PASJ, 59, 61

Okuda, T., Kohno, K., Iguchi, S., \& Nakanishi, K. 2005, ApJ, 620, 673

Owen, F. N., O'Dea, C. P., \& Keel, W. C. 1990, ApJ, 352, 44

Phillips, M. M., Jenkins, C. R., Dopita, M. A., Sadler, E. M., \& Binette, L. 1986, AJ, 91, 1062

Pringle, J. E. 1981, ARA\&A, 19, 137

Prugniel, P., \& Simien, F. 1997, A\&A, 321, 111

Ravindranath, S., Ho, L. C., Peng, C. Y., Filippenko, A. V., \& Sargent, W. L. W. 2001, AJ, 122, 653

Rest, A., van den Bosch, F. C., Jaffe, W., Tran, H., Tsvetanov, Z., Ford, H. C., Davies, J., \& Schafer, J. 2001, AJ, 121, 2431

Sadler, E. M., Oosterloo, T. A., Morganti, R., \& Karakas, A. 2000, AJ, 119,1180

Sage, L. J., Welch, G. A., \& Young, L. M. 2007, ApJ, 657, 232

Schawinski, K., et al. 2006, Nature, 442, 888

Schaye, J. 2004, ApJ, 609, 667

Sérsic, J. L. 1968, Atlas de galaxias australes (Cordoba, Argentina: Observatorio Astronomico)

Shakura, N. I., \& Sunyaev, R. A. 1973, A\&A, 24, 337

Silk, J., \& Rees, M. J. 1998, A\&A, 331, L1

Springel, V., Di Matteo, T., \& Hernquist, L. 2005, ApJ, 620, L79

Stiavelli, M., Miller, B. W., Ferguson, H. C., Mack, J., Whitmore, B. C., \& Lotz, J. M. 2001, AJ, 121, 1385

Tan, J. C., Beuther, H., Walter, F., \& Blackman, E. G. 2007, ApJ, in press

Tan, J. C., \& Blackman, E. G. 2005, MNRAS, 362, 983

Terzić, B., \& Graham, A. W. 2005, MNRAS, 362, 197

Tomita, A., Aoki, K., Watanabe, M., Takata, T., \& Ichikawa, S.-i. 2000, AJ, 120, 123

Toomre, A. 1964, ApJ, 139, 1217

Tran, H. D., Tsvetanov, Z., Ford, H. C., Davies, J., Jaffe, W., van den Bosch, F. C., \& Rest, A. 2001, AJ, 121, 2928

Trujillo, I., Burkert, A., \& Bell, E. F. 2004a, ApJ, 600, L39

Trujillo, I., Erwin, P., Asensio Ramos, A., \& Graham, A. W. 2004b, AJ, 127, 1917

van Dokkum, P. G., \& Franx, M. 1995, AJ, 110, 2027

Vila-Vilaró, B., Cepa, J., \& Butner, H. M. 2003, ApJ, 594, 232

Wada, K. 2004, in Carnegie Observatories Astrophysics Series,

Vol. 1: Coevolution of Black Holes and Galaxies, ed. L. C. Ho (Cambridge: Cambridge Univ. Press), 186

Wada, K., \& Tomisaka, K. 2005, ApJ, 619, 93

Wiklind, T., Combes, F., \& Henkel, C. 1995, A\&A, 297, 643

Wiklind, T., \& Henkel, C. 1995, A\&A, 297, L71+

Yi, S. K., et al. 2005, ApJ, 619, L111

Young, L. M. 2002, AJ, 124, 788

- 2005, ApJ, 634, 258 